\title{
The Chandra LETGS high resolution X-ray spectrum of the isolated neutron star RX J1856.5-3754
}

\author{
V. Burwitz ${ }^{1}$, V. E. Zavlin ${ }^{1}$, R. Neuhäuser ${ }^{1}$, P. Predehl${ }^{1}$, J. Trümper ${ }^{1}$, and A. C. Brinkman ${ }^{2}$ \\ 1 Max-Planck-Institut für extraterrestrische Physik, PO Box 1312, 85741 Garching, Germany \\ 2 Space Research Organization of the Netherlands, Soorbonnelaan 2, 3584 CA Utrecht, The Netherlands
}

Received 1 August 2001 / Accepted 18 September 2001

\begin{abstract}
We present the Chandra LETGS X-ray spectrum of the nearby ( $\simeq 60 \mathrm{pc})$ neutron star RXJ1856.53754. Detailed spectral analysis of the combined X-ray and optical data rules out the nonmagnetic neutron star atmosphere models with hydrogen, helium, iron and solar compositions. We also conclude that strongly magnetized atmosphere models are unable to represent the data. The data can be explained with a two-component blackbody model. The harder component with temperature of $k T_{\mathrm{bb}}^{\infty} \simeq 63 \mathrm{eV}$ and a radius $R_{\mathrm{bb}}^{\infty} \simeq 2.2 \mathrm{~km}$ of the emitting region well fits the X-ray data and can be interpreted as radiation from a hot region on the star's surface.
\end{abstract}

Key words. stars: atmospheres - stars: individual: RX J1856.5-3754 - stars: neutron - X-rays: stars

\section{The neutron star RXJ1856.5-3754}

It was suggested by Walter et al. (1996) and supported by Neuhäuser et al. (1997) that RXJ1856.5-3754 (or RXJ1856 for short) is an isolated neutron star (NS). It has a strong and non-variable $\mathrm{X}$-ray flux $\simeq 1.5 \times$ $10^{-11} \mathrm{erg} \mathrm{s}^{-1} \mathrm{~cm}^{-2}$ and a soft spectrum with a blackbody temperature $k T_{\mathrm{bb}}^{\infty} \simeq 57 \mathrm{eV}$. Thousands of such NSs were expected in RASS (e.g., Colpi et al. 1993; Madau \& Blaes 1994), moving fast through the interstellar medium. Only a few such objects have been found, three of them show pulsations on time-scale of seconds (Haberl et al. 1997, 1999; Hambaryan et al. 2001). Neuhäuser \& Trümper (1999) argued that the number of isolated NSs expected in RASS was overestimated mainly due to unrealistic velocity distributions.

Walter \& Matthews (1997) and Neuhäuser et al. (1998) found an optical counterpart for RXJ1856 with $V \simeq 26 \mathrm{mag}$. This and the large proper motion of $\simeq 0.33 \mathrm{mas} \mathrm{yr}^{-1}$ (Walter 2001; Neuhäuser 2001) are additional arguments that it is indeed an isolated NS. Walter (2001) also detected parallactic motion, determined the distance to the source $d=61_{-8}^{+9} \mathrm{pc}$ and suggested that RXJ1856 could be the remnant of the original primary of the $\zeta$ Oph system. This implies a NS age of $\sim 10^{6} \mathrm{yr}$.

First spectral modelling of RXJ1856 based on its ROSAT data was presented by Pavlov et al. (1996; hereafter P96), who showed that the optical/UV flux predicted by NS atmosphere models depends drastically on the

Send offprint requests to: V. Burwitz, e-mail: burwitz@mpe.mpg.de surface chemical composition. More recently Pons et al. (2002; hereafter P02) have analyzed combined optical and $\mathrm{X}$-ray data and concluded that it may have either a $\mathrm{Fe}$ or Si-ash atmosphere. However, the inferred NS radius $R \approx 6 \mathrm{~km}$ and mass $M \approx 0.9 M_{\odot}$ are not allowed for any plausible equation of state of the NS inner matter.

RXJ1856 was observed with the Chandra Low Energy Transmission Grating Spectrometer (LETGS; Brinkman et al. 2000). First preliminary results were presented by Burwitz et al. (2001). Here we describe the LETGS data (Sect. 2), results of spectral (Sect. 3) and timing (Sect. 4) analysis and discuss implications on the nature of RXJ1856 (Sect. 5).

\section{LETGS data extraction}

RXJ1856 was observed on March 10, 2000 with the standard LETGS (LETG + HRC-S) configuration in a $56.1 \mathrm{ks}$ exposure. The LETGS spectrum of RXJ1856 was extracted from the level 1.5 event file. The only pulse-height filtering applied to the data was the removal of photons with a pulse-height amplitude equal to 255 as other pulseheight filters do not reduce the background level evenly for all energies. We used the extraction region recommended in the Chandra Proposers' Observatory Guide ${ }^{1}$ [POG]) for extracting the source spectrum (see Fig. 1). For the background, large regions above and below the source extraction area are selected. Towards longer wavelengths $\lambda>48.4 \AA$ the background regions become wider

\footnotetext{
1 http://asc.harvard.edu/udocs/docs/docs.html
} 
in order to maintain the constant ratio of areas between source and background spectral bins (here the ratio is equal to 6 ). The measured dispersed source count rate is $290 \pm 6 \mathrm{ks}^{-1}$ in the $0.15-0.82 \mathrm{keV}$ range (where the source spectrum prevails over the background). The extracted source spectrum (binned in 686 spectral bins) and the most up-to-date effective area tables for the 1st order (status of October 31, 2000) ${ }^{2}$ were used for spectral fits.

\section{Spectral analysis}

To search for spectral lines, we used the method applied by Pavlov et al. (2001) to the dispersed LETGS data on the Vela pulsar. We first binned the extracted source-plusbackground and background spectra in $0.02 \AA$ bins. Then we grouped 10-20 sequent bins and estimated the deviation of source counts in each bin of a given group from the mean value in the group. This method revealed maximum deviation at a $2.9 \sigma$ level.

Since there exist the ROSAT PSPC data ( $\sim 20200$ counts collected in a $6.3 \mathrm{ks}$ exposure), we first checked whether the LETGS and PSPC data yield consistent results in spectral fits. The blackbody fits showed that the allowed domains of the fitting parameters do not formally overlap with each other: $k T_{\mathrm{bb}}^{\infty}=57 \pm 3 \mathrm{eV}, R_{\mathrm{bb}}^{\infty}=(3.7 \pm 0.7) d_{60} \mathrm{~km}\left(d_{60}=d / 60 \mathrm{pc}\right)$ and $n_{\mathrm{H}, 20}=n_{\mathrm{H}} /\left(10^{20} \mathrm{~cm}^{-2}\right)=1.47 \pm 0.25$ (the uncertainties are given at a $3 \sigma$ level) with $\chi_{\nu}^{2}=1.5$ for the PSPC data, and $k T_{\mathrm{bb}}^{\infty}=63 \pm 3 \mathrm{eV}, R_{\mathrm{bb}}^{\infty}=(2.2 \pm 0.3) d_{60} \mathrm{~km}$ and $n_{\mathrm{H}, 20}=1.03 \pm 0.20\left(\chi_{\nu}^{2}=1.0\right)$ for the LETGS data. The difference between the blackbody models given by the two fits (see Fig. 2) is most likely attributed to uncertainties in the calibration of LETGS and PSPC.

The next step in analysing the LETGS spectrum is to apply NS atmosphere models. The results of P96 and P02 showed that the light element, $\mathrm{H}$ and He, nonmagnetic atmosphere models can be firmly ruled out because the models (i) yield too small distances to RXJ1856, $d \simeq(5-6)(R / 10 \mathrm{~km}) \mathrm{pc}$ and (ii) overpredict the optical flux by a factor of 20-30. P02 found that heavier element atmosphere models can be reconciled with the PSPC and optical data at $k T=57.4 \mathrm{eV}, R=6.0 d_{60} \mathrm{~km}$ for pure $\mathrm{Fe}$, and $k T=58.7 \mathrm{eV}, R=6.0 d_{60} \mathrm{~km}$ for Si-ash compositions, assuming the gravitational parameter $g_{\mathrm{r}}=\left[1-R_{\mathrm{g}} / R\right]^{1 / 2}=0.77\left(R_{\mathrm{g}}=2 G M / c^{2}\right.$ is the Schwarzschild radius $)^{3}$. Varying $g_{\mathrm{r}}$ resulted in negligible changes in the inferred model parameters unless the strong absorption line at (unredshifted) $E_{\text {true }} \simeq 0.79 \mathrm{keV}$ (due to absorption by FexVIII ions) penetrates in lower (redshifted) energies $E=g_{\mathrm{r}} E_{\text {true }}<0.6 \mathrm{keV}$ and significantly affects the quality of the PSPC spectral fits. The effects of gravity on the emergent spectra are too small to affect the results of the spectral fits. The fit of the PSPC spectrum with Fe atmosphere models of Pavlov \& Zavlin (2000) yields $d=54 \pm 10 \mathrm{pc}, k T=45 \pm 5 \mathrm{eV}$ at $R=10 \mathrm{~km}$

\footnotetext{
2 http://asc.harvard.edu/cal/Links/Letg/User

3 For the atmosphere model fits we give parameters as measured at the NS surface, $T=g_{\mathrm{r}}^{-1} T^{\infty}$ and $R=g_{\mathrm{r}} R^{\infty}$.
}

and $M=1.4 M_{\odot}$, that are well consistent with those obtained by P02. Although the LETGS spectrum revealed no statistically significant features, it is worthwhile trying the heavy element atmosphere models on these data. To fit the LETGS data, we fixed the distance at $d=60 \mathrm{pc}$ and obtained best fitting parameters $n_{\mathrm{H}, 20}=1.72, k T=40.3 \mathrm{eV}$, $R=12.0 d_{60} \mathrm{~km}$ at $M=1.4 M_{\odot}$ (see red curves in the left panels of Fig. 3). The model fits well at (redshifted) $E \lesssim 0.5 \mathrm{keV}$ where the atmosphere spectrum shows no strong spectral features. However, the situation is drastically different at the higher energies because of the strong absorption line at $E=0.81 E_{\text {true }} \simeq 0.64 \mathrm{keV}$ (see above) of a characteristic width $\sim 70 \mathrm{eV}$ and a weaker absorption complex at $E \simeq 0.55-0.58 \mathrm{keV}$. We note that the LETGS spectrum shows a strong instrumental feature at $E=$ $0.61-0.63 \mathrm{keV}$ (POG). Despite of a formally small value of $\chi_{\nu}^{2}=1.2$ obtained in this fit, the deviation between the model and LETGS spectra at $E \gtrsim 0.5 \mathrm{keV}$ are large enough to regard the fit as unacceptable. Increasing $g_{\mathrm{r}}$ (i.e. reducing the redshift) does not improve the fit because the absorption lines remain within the observed energy range (see an example of the spectral fit at $M=0.2 M_{\odot}$, $R=9.8 \mathrm{~km}$ and $g_{\mathrm{r}}=0.97$ in the upper left panel of Fig. 3 [blue curve]).

The same strong Fe features are expected to be present in the Si-ash model spectra as Fe composes $68 \%$ of the ash (P02). Therefore, the situation with the Si-ash model fit to the LETGS spectrum should be similar to the Fe case. We also applied atmosphere models with a solar composition containing 2\% heavy elements (Grevesse \& Noels 1993). The model spectra show numerous prominent spectral features (mainly due to absorption by $\mathrm{Fe}, \mathrm{C}$ and $\mathrm{O}$ ions) in the whole observed energy range (see right panels in Fig. 3, and also Rajagopal \& Romani 1996) which are inconsistent with the LETGS data.

\section{Timing analysis}

For the timing analysis we used 11606 counts extracted from a $2^{\prime \prime}$-radius circle centered at the zero-order image (we did not use the dispersed data as they are strongly contaminated by background). We had to restrict the search for a periodical signal at the (upper) frequency $f=40 \mathrm{~Hz}$ because of the time-tag problem (see POG), unrecovered in this observation. As a result, the HRC timing accuracy in this observation was about $4-5 \mathrm{~ms}$ (vs. $16 \mu \mathrm{s}$ planned). We applied the standard $Z_{1}^{2}$ (Rayleigh) test (Buccheri et al. 1983) as well as the method based on the Bayesian statistics (Gregory \& Loredo 1996; Zavlin et al. 2000; Hambaryan et al. 2001). The frequency range $f=10^{-3}-40 \mathrm{~Hz}\left(P=25 \mathrm{~ms}-10^{3} \mathrm{~s}\right)$ revealed no statistically significant signals (maximum significance is at a $2.1 \sigma$ level). Assuming a sinusoidal signal, we put an upper limit $f_{\mathrm{p}}<8 \%$ on its pulsed fraction.

\section{Discussion and summary}

The most striking result of our Chandra LETGS data analysis (extended to the optical range $[\mathrm{P} 96 ; \mathrm{P} 02]$ ) is 


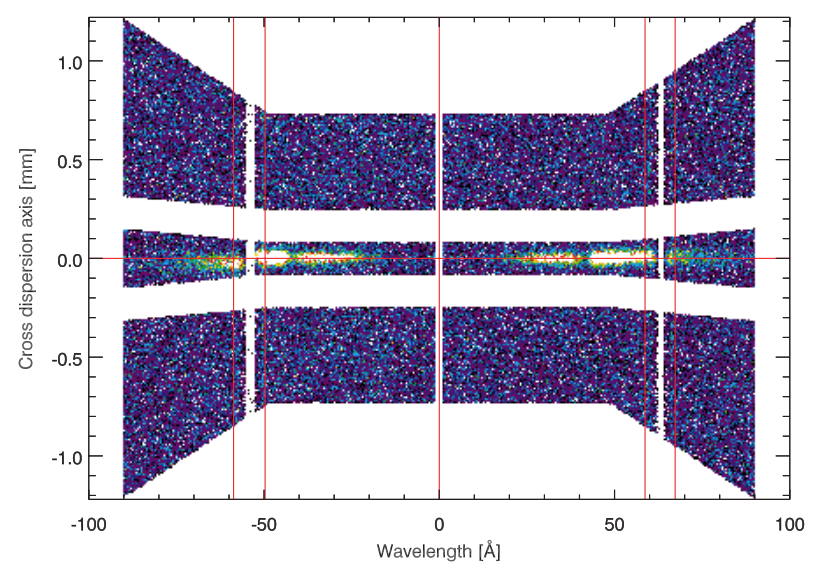

Fig. 1. Regions used for extracting the source and background dispersed spectra. The vertical red lines on either side of the gaps between the HRC-S detector plates indicate the the areas that are masked out to avoid getting artificial spectral features due to the dithering across the plate edges (see POG).

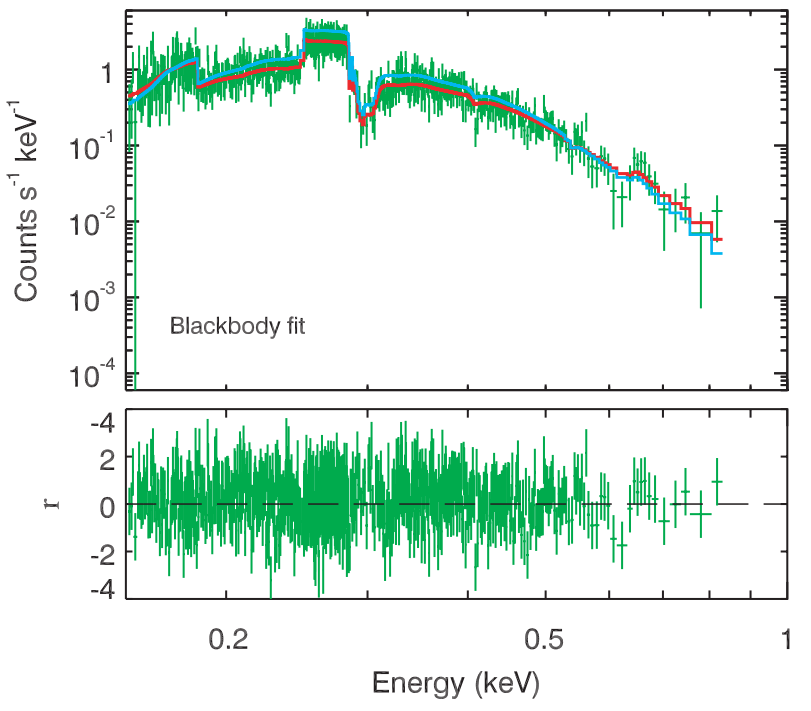

Fig. 2. The best blackbody fit to the LETGS spectrum (red curve) with the blackbody model given by the best PSPC fit (blue curve).
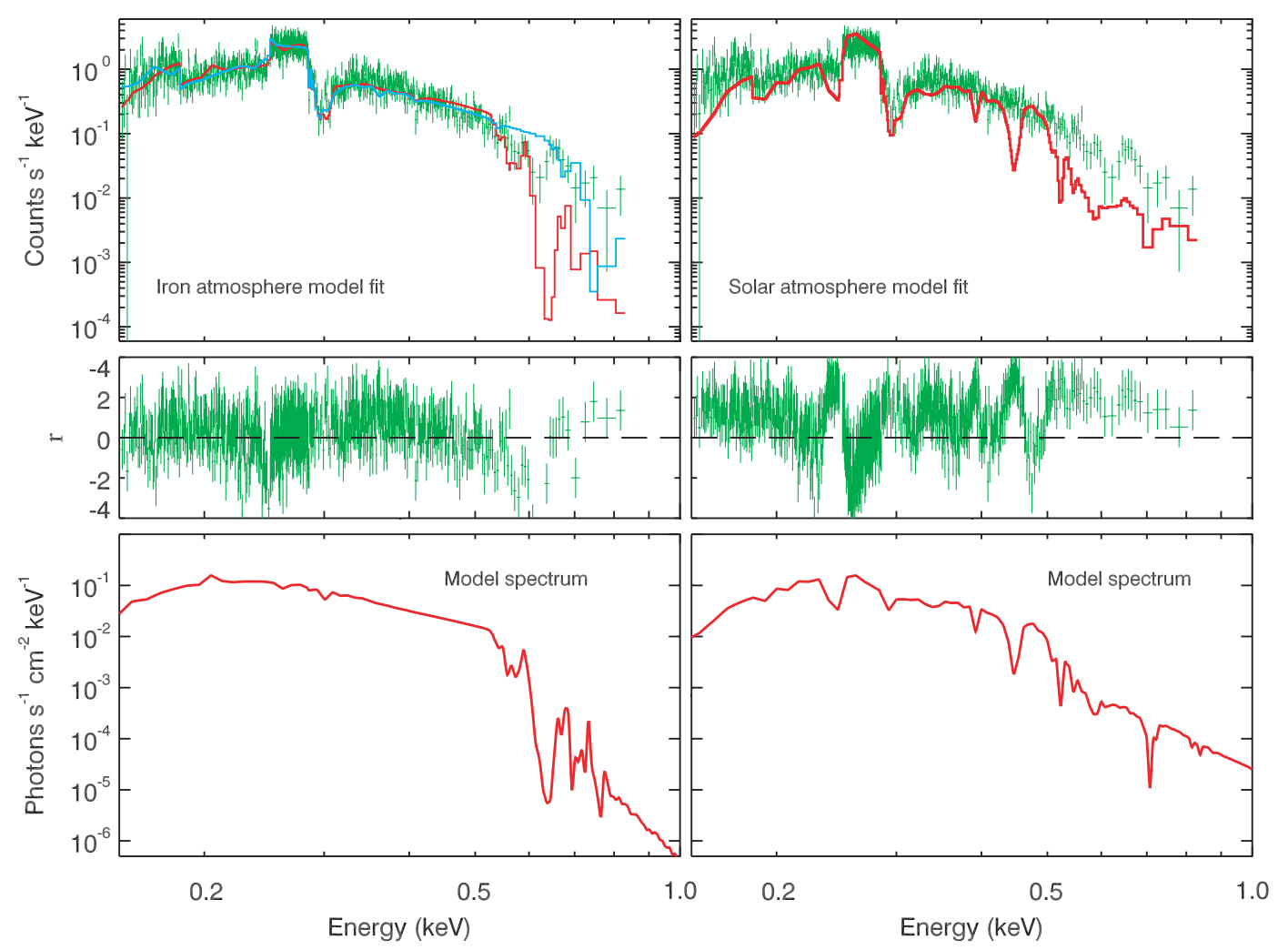

Fig. 3. The LETGS spectrum fitted with the Fe (left) and solar mixture (right) atmosphere models (see text for details).

the fact that a simple blackbody model yields a much better fit to the RXJ1856 spectrum than either light or heavy element nonmagnetic atmosphere models. Note that the heavy element models are inconsistent with the high-resolution LETG spectrum. On the other hand, nonmagnetic $\mathrm{H} / \mathrm{He}$ models do not work either because they predict to high optical fluxes. A remedy may be provided if RXJ1856 has a strong magnetic field as indicated by a bow-shock nebula seen in $\mathrm{H}_{\alpha}$ optical observations (van Kerkwijk \& Kulkarni 2000). Similar to the case with the nonmagnetic models, the magnetized $\mathrm{H}$ atmosphere fits result in a too small distance and a too high optical flux (P96). Magnetized Fe atmosphere spectra at $k T \sim 60 \mathrm{eV}$ (Rajagopal et al. 1997) reveal a lot of strong absorption features in the $0.1-1.0 \mathrm{keV}$ range which should have been resolved with the LETGS spectral resolution. Hence, we conclude that the available NS atmosphere models cannot represent both the X-ray and optical data on RXJ1856. 
Regardless of the chemical composition, radiation emitted from the surface of a magnetized NS should exhibit spectral lines of the electron (at $E_{B \mathrm{e}}=11.6 B_{12} \mathrm{keV}$ ) and proton (at $E_{B \mathrm{p}}=6.3 B_{12} \mathrm{eV}$ ) cyclotron resonances ( $B_{12}$ is the strength of magnetic field in units of $10^{12} \mathrm{G}$ ). The lack of significant features in the LETGS spectrum $(0.15-0.82 \mathrm{keV})$ may appear to exclude the magnetic fields $B \simeq(1.3-7.0) \times 10^{10} \mathrm{G}$ and $B \simeq(0.2-1.3) \times 10^{14} \mathrm{G}$. But we note that these restrictions on $B$ are not very stringent because these lines may be rather faint if the surface layers are only weakly ionized. Besides, nonuniformity of the magnetic field over the surface should lead to a strong smearing of the lines (Zavlin et al. 1995).

Remarkably, the X-ray spectrum of the other isolated NS, RX J0720.4-3125, obtained with XMM-Newton also shows no significant spectral features and is well fitted with a blackbody model of $k T_{\mathrm{bb}}^{\infty} \simeq 86 \mathrm{eV}$ (Paerels et al. 2001).

P02 argued that a two-component blackbody model can be reconciled with both the optical and X-ray data on RXJ1856. In this model the hard component of $k T^{\infty} \simeq$ $55 \mathrm{eV}$ is emitted from a $\sim 20 \%$ fractional area on the NS surface and fits the X-ray data, whereas the soft component of $k T^{\infty} \simeq 20 \mathrm{eV}$ represents radiation from the cool surface and matches the optical data. The required non-uniform distribution of the surface temperature may be due to the strong dependance of thermal conductivity of the NS crust on the magnetic field at $B \gtrsim 10^{12} \mathrm{G}$ (Greenstein \& Hartke 1983). At temperatures $k T \lesssim 86 \mathrm{eV}$ and magnetic fields $B \gtrsim 10^{13} \mathrm{G}$ hydrogen (if present on the surface) can be in form of polyatomic molecules and/or a condensed liquid (Lai \& Salpeter 1997). Although, to our knowledge, no reliable calculations have been done, one may speculate that such a condensed matter surface emits radiation close to the blackbody spectrum at a temperature close to that of the surface, as suggested by Pavlov $(2000)^{4}$. Then, the two-component blackbody interpretation may be considered as a simplified model of the thermal radiation from such a magnetized and relatively cool NS.

New important information for elucidating the nature of RXJ1856 is expected to come from the forthcoming $50 \mathrm{ks} X M M$-Newton and $450 \mathrm{ks}$ Chandra observations. They will yield an order of magnitude more counts in the dispersed spectra than in the data presented above. This will allow one to take more advantage of the high spectral resolution. Additionally, the new data would enable an unambiguous detection of X-ray pulsations from the source even if the pulsed fraction is as low as $3 \%$. Once the period is found in the new data, it could be traced back to the earlier ROSAT and Chandra data. This would give estimates on the period derivative and, as consequence, magnetic field and age of RXJ1856. The latter, compared with the estimate derived from the optical observations, would shed more light on the nature of this enigmatic source.

\footnotetext{
${ }^{4}$ http://online.itp.ucsb.edu/online/neustars00/ pavlov
}

Acknowledgements. We thank Fred Walter, George Pavlov and Frits Paerels for intensive discussions. This research was supported by Deutsches Zentrum für Luft- und Raumfahrt grant 50 OX 0001.

\section{References}

Buccheri, R., Bennett, K., Bignami, G. F., et al. 1983, A\&A, 128, 245

Brinkman, A. C., Gunsing, C. J. T., Kaastra, J. S., et al. 2000, ApJ, 530, L111

Burwitz, V., Dennerl, K., Haberl, F., et al. 2001, in X-ray Astronomy 2000, ed. R. Giacconi, L. Stella, \& S. Serio, ASP Conf. Ser., 234, in press

Colpi, M., Campana, S., \& Treves, A. 1993, A\&A, 278, 161

Gregory, P. C., \& Loredo, T. J. 1996, ApJ, 473, 1059

Greenstein, G., \& Hartke, G. J. 1983, ApJ, 271, 283

Grevesse, N., \& Noels, A. 1993, in Origin and Evolution of the Elements, ed. E. Prantzos, M. Vaugioni-Flam, \& M. Gasse (Cambridge Univ. Press), 14

Haberl, F., Motch, C., Buckley, D. A. H., Zickgraf, F.-J., \& Pietsch, W. 1997, A\&A, 326, 662

Haberl, F., Pietsch, W., \& Motch, C. 1999, A\&A, 351, L53

Hambaryan, V., Hasinger, G., Schwope, A. D., \& Schulz, N. S. 2001, A\&A, in press

van Kerkwijk, M., \& Kulkarni, S. 2000, ESO press release 19/00

Lai, D., \& Salpeter, E. E. 1997, ApJ, 491, 270

Madau, P., \& Blaes, O. 1994, ApJ, 423, 748

Neuhäuser, R. 2001, AN, 322, 3

Neuhäuser, R., \& Trümper, J. 1999, A\&A, 143, 151

Neuhäuser, R., Thomas, H.-C., Danner, R., Peschke, S., \& Walter, F. M. 1997, A\&A, 318, L43

Neuhäuser, R., Thomas, H.-C., \& Walter, F. M. 1998, The Messenger, 92, 27

Paerels, F., Mori, K., Motch, C., et al. 2001, A\&A, 365, L298

Pavlov, G. G. 2000, Talk at the ITP/UCSB workshop, Spin and Magnetism of Young Neutron Stars

Pavlov, G. G., \& Zavlin, V. E. 2000, in Highly Energetic Physical Processes and Mechanisms for Emission from Astrophysical Plasmas, ed. P. C. H. Martens, S. Tsuruta, \& M. A. Weber, IAU Symp., 195, 103

Pavlov, G. G., Zavlin, V. E., Trümper, J., \& Neuhäuser, R. 1996, ApJ, 472, L33 (P96)

Pavlov, G. G., Zavlin, V. E., Sanwal, D., Burwitz, V., \& Garmire, G. P. 2001, ApJ, 552, L129

Pons, J. A., Walter, F. M., Lattimer, J. M., et al. 2002, ApJ, 564 , in press (P02)

Rajagopal, M., \& Romani, R. W. 1996, ApJ, 461, 327

Rajagopal, M., Romani, R. W., \& Miller, M. C. 1997, ApJ, 479,347

Walter, F. M., \& Matthews, L. D. 1997, Nature, 389, 358

Walter, F. M., Wolk, S. J., \& Neuhäuser, R. 1996, Nature, 379, 233

Walter, F. M. 2001, ApJ, 549, 433

Zavlin, V. E., Pavlov, G. G., Shibanov, Yu. A., \& Ventura, J. 1995, A\&A, 297, 441

Zavlin, V. E., Pavlov, G. G., Sanwal, D., \& Trümper, J. 2000, ApJ, 540, L25 